Pacific

Journal of

Mathematics

CONFORMAL HOLONOMY EQUALS AMBIENT HOLONOMY

ANDREAS ČAP, A. ROD GOVER,

C. Robin GRAHAM AND MATTHIAS HAMMERL 


\title{
CONFORMAL HOLONOMY EQUALS AMBIENT HOLONOMY
}

\author{
ANDREAS ČAP, A. ROD GOVER, \\ C. Robin Graham AND MATTHias HAMMERL
}

\begin{abstract}
We study the relation between two notions of holonomy on a conformal manifold. The first is the conformal holonomy, defined to be the holonomy of the normal tractor connection. The second is the holonomy of the FeffermanGraham ambient metric of the conformal manifold. It is shown that the infinitesimal conformal holonomy and the infinitesimal ambient holonomy always agree up to the order that the ambient metric is defined.
\end{abstract}

\section{Introduction}

The tractor bundle $\mathcal{T}$ of a smooth conformal manifold $(M, c)$ of dimension $n \geq 3$ and signature $(p, q), p+q=n$, is a rank- $(n+2)$ vector bundle naturally associated to the conformal structure, which carries a canonical connection $\nabla$; see [Bailey et al. 1994]. This connection is characterized by a normalization condition on its curvature, whence it is called the normal tractor connection [Čap and Gover 2003]. It can be viewed as a conformally invariant analog of the Levi-Civita connection of a Riemannian manifold and has played an essential role in many recent developments in conformal geometry. The holonomy of $(\mathcal{T}, \nabla)$ is called the conformal holonomy of $(M, c)$. Following early work [Armstrong 2007; Leistner 2006; Leitner 2005], its study has been the focus of active recent research; see, e.g., [Alt 2012; Armstrong and Leitner 2012; Lischewski 2015].

Another invariant object associated to a conformal manifold is the ambient metric of [Fefferman and Graham 1985; 2012]. This is a smooth pseudo-Riemannian metric of signature $(p+1, q+1)$ on a space of dimension $n+2$, determined up to diffeomorphism along a canonical hypersurface, to infinite order if $n$ is odd, and to order $\frac{n}{2}-1$ if $n$ is even. Its Levi-Civita connection is another connection associated to the conformal manifold and one can also consider its holonomy. Because the

Čap and Gover gratefully acknowledge support from the Royal Society of New Zealand via Marsden Grant 13-UOA-018. Further, support by projects P23244-N13 (Čap and Hammerl) and P27072-N25 (Čap) of the Austrian Science Fund (FWF) is gratefully acknowledged. Research of Graham is partially supported by NSF grant \# DMS 1308266.

MSC2010: primary 53A30; secondary 53C29.

Keywords: holonomy, ambient metric, tractor, conformal geometry. 
holonomy group of a connection is a global invariant and the ambient metric is only invariantly defined as a jet along a hypersurface, its holonomy group is not the appropriate object to study. Instead we consider the infinitesimal holonomy, which depends only on the jet at a point. The main result of this paper asserts that, suitably interpreted, the infinitesimal holonomies of the tractor connection and the Levi-Civita connection of the ambient metric agree at each point.

In order to formulate the result precisely, we describe a realization of the tractor bundle in ambient terms which was derived in [Čap and Gover 2003]. Details will be provided in Section 2. If $(M, c)$ is a conformal manifold, its metric bundle is the ray bundle $\mathcal{G} \subset S^{2} T^{*} M$ whose sections are the metrics $g \in c$. The ambient space is $\mathcal{G} \times \mathbb{R}$, in which $\mathcal{G}$ is embedded as the hypersurface $\mathcal{G} \times\{0\}$. There are dilations $\delta_{s}: \mathcal{G} \rightarrow \mathcal{G}$ given by $\delta_{s}\left(x, g_{x}\right)=\left(x, s^{2} g_{x}\right), s>0$, which extend to $\mathcal{G} \times \mathbb{R}$ acting in the first factor. For $x \in M$, we denote by $\mathcal{G}_{x}$ the fiber of $\mathcal{G}$ over $x$, and we view $\mathcal{G}_{x}$ as a 1-dimensional submanifold of $\mathcal{G} \times \mathbb{R}$ via $\mathcal{G}_{x} \subset \mathcal{G}=\mathcal{G} \times\{0\} \subset \mathcal{G} \times \mathbb{R}$. Then $\left.T(\mathcal{G} \times \mathbb{R})\right|_{\mathcal{G}_{x}}$ denotes the tangent bundle to $\mathcal{G} \times \mathbb{R}$ restricted to the submanifold $\mathcal{G}_{x}$, a rank- $(n+2)$ vector bundle over $\mathcal{G}_{x}$. The standard tractor bundle of $(M, c)$ can be realized as the rank- $(n+2)$ vector bundle $\mathcal{T} \rightarrow M$ with fiber

$$
\mathcal{T}_{x}=\left\{U \in \Gamma\left(\left.T(\mathcal{G} \times \mathbb{R})\right|_{\mathcal{G}_{x}}\right):\left(\delta_{s}\right)^{*} U=s^{-1} U, s>0\right\} .
$$

The right-hand side of (1-1) is clearly a vector space of dimension $n+2$ varying smoothly with $x$. A section of $\mathcal{T}$ on $M$ is thus a vector field in $\mathcal{G} \times \mathbb{R}$ defined on $\mathcal{G}$ which is homogeneous of degree -1 with respect to the $\delta_{s}$.

As we will also review in Section 2, an ambient metric for $(M, c)$ is a pseudoRiemannian metric $\tilde{g}$ which is defined in a dilation-invariant neighborhood $\widetilde{\mathcal{G}}$ of $\mathcal{G}$ in $\mathcal{G} \times \mathbb{R}$ by certain conditions. As indicated above, it is uniquely determined by $(M, c)$ up to diffeomorphism to infinite order if $n$ is odd and to order $\frac{n}{2}-1$ if $n$ is even.

It seems that the notion of infinitesimal holonomy was first introduced and studied systematically by Nijenhuis [1953a; 1953b; 1954]. A standard reference is [Kobayashi and Nomizu 1963]. If $(\mathcal{V}, \nabla)$ is a smooth vector bundle with connection on a manifold $M$ and $x \in M$, the infinitesimal holonomy algebra $\mathfrak{h o l}_{x}$ of $(\mathcal{V}, \nabla)$ at $x$ is the subspace of End $\mathcal{V}_{x}$ defined by

$$
(1-2) \mathfrak{h o l}_{x}=\operatorname{span}_{\mathbb{R}}\left\{\nabla_{\eta_{k}} \nabla_{\eta_{k-1}} \ldots \nabla_{\eta_{\mathcal{3}}}\left(R\left(\eta_{1}, \eta_{2}\right)\right)(x): k \geq 2, \eta_{1}, \ldots, \eta_{k} \in \mathfrak{X}(M)\right\} .
$$

Here $\mathfrak{X}(M)$ denotes the space of smooth vector fields on $M$ and $R: \Lambda^{2} T M \rightarrow$ End $\mathcal{V}$ the curvature of $\nabla$. It is a standard fact that $\mathfrak{h o l}_{x}$ is a subalgebra of End $\mathcal{V}_{x}$ for its natural Lie algebra structure with bracket the commutator of endomorphisms. Clearly $\mathfrak{h o l}{ }_{x}$ depends only on the infinite order jet of $\nabla$ at $x$, and so in particular there is generally no relation between $\mathfrak{h o l}_{x}$ and $\mathfrak{h o l}_{y}$ for $x \neq y$. However, if $M$ and $(\mathcal{V}, \nabla)$ are real-analytic, then $\mathfrak{h o l}_{x}$ is the Lie algebra of $\operatorname{Hol}_{x}$, where $\operatorname{Hol}_{x} \subset$ Aut $\mathcal{V}_{x}$ is the usual holonomy group of $(\mathcal{V}, \nabla)$ defined by parallel translation around loops 
based at $x$. Of course, $\mathrm{Hol}_{x}$ is always isomorphic to $\mathrm{Hol}_{y}$ for $M$ smooth and connected.

For a conformal manifold $(M, c)$, we denote by $\mathfrak{h o l}_{x}$ the infinitesimal holonomy at $x$ of $(\mathcal{T}, \nabla)$, where $\nabla$ is the normal tractor connection. Thus $\mathfrak{h o l}_{x}$ is a subalgebra of End $\mathcal{T}_{x}$. The realization (1-1) of $\mathcal{T}_{x}$ induces the realization

$$
\text { End } \mathcal{T}_{x}=\left\{E \in \Gamma\left(\left.\operatorname{End} T(\mathcal{G} \times \mathbb{R})\right|_{\mathcal{G}_{x}}\right):\left(\delta_{s}\right)^{*} E=E, s>0\right\}
$$

of End $\mathcal{T}_{x}$. Thus an element of $\mathfrak{h o l}_{x}$ is realized as a section of the vector bundle End $\left.T(\mathcal{G} \times \mathbb{R})\right|_{\mathcal{G}_{x}}$ over $\mathcal{G}_{x}$ which is homogeneous of degree 0 with respect to the $\delta_{s}$. For any $z \in \mathcal{G}_{x}$, evaluation at $z$ is an isomorphism

$$
\mathrm{ev}_{z}: \text { End } \mathcal{T}_{x} \rightarrow \text { End } T_{z}(\mathcal{G} \times \mathbb{R})
$$

So $\mathrm{ev}_{z}\left(\mathfrak{h o l}_{x}\right)$ is an isomorphic copy of $\mathfrak{h o l}_{x}$ in End $T_{z}(\mathcal{G} \times \mathbb{R})$.

If $\tilde{g}$ is an ambient metric for $(M, c)$ and $x \in M$, the infinitesimal holonomy at $z \in \mathcal{G}_{x}$ of the Levi-Civita connection $\widetilde{\nabla}$ of $\tilde{g}$ is a subalgebra of End $T_{z} \widetilde{\mathcal{G}}=\operatorname{End} T_{z}(\mathcal{G} \times \mathbb{R})$. If $n$ is odd, we denote this subalgebra $\widetilde{\mathfrak{h o l}}_{z}$. This is clearly independent of the infinite-order ambiguity in $\tilde{g}$. However, when $n$ is even, the ambient metric is determined by $(M, c)$ only to order $\frac{n}{2}-1$ along $\mathcal{G}$. So we need to restrict the number of differentiations transverse to $\mathcal{G}$ to avoid this ambiguity. Therefore, when $n \geq 4$ is even, we define

$$
(1-4) \quad \widetilde{h o l}_{z}=\operatorname{span}_{\mathbb{R}}\left\{\widetilde{\nabla}_{\tilde{\xi}_{k}} \widetilde{\nabla}_{\tilde{\xi}_{k-1}} \ldots \widetilde{\nabla}_{\tilde{\xi}_{3}}\left(\widetilde{R}\left(\tilde{\xi}_{1}, \tilde{\xi}_{2}\right)\right)(z): k \geq 2, \tilde{\xi}_{1}, \ldots, \tilde{\xi}_{k} \in \mathfrak{X}(\widetilde{\mathcal{G}})\right\},
$$

where $\widetilde{R}$ is the curvature of $\widetilde{\nabla}$, but we impose the requirement that no more than $\frac{n}{2}-2$ of the vector fields $\tilde{\xi}_{1}, \ldots, \tilde{\xi}_{k}$ are somewhere transverse to $\mathcal{G}$. Then $\widetilde{\nabla}_{\tilde{\xi}_{k}} \widetilde{\nabla}_{\tilde{\xi}_{k-1}} \ldots \widetilde{\nabla}_{\tilde{\xi}_{3}}\left(\widetilde{R}\left(\tilde{\xi}_{1}, \tilde{\xi}_{2}\right)\right)$ depends on at most $\frac{n}{2}-1$ transverse derivatives of $\tilde{g}$, so its value at $z$ is independent of the ambiguity at order $\frac{n}{2}$. A priori, (1-4) is only defined as a vector space, but it is a consequence of Theorem 1.1 that it is a Lie subalgebra of End $T_{z} \widetilde{\mathcal{G}}$.

Our main result is the following.

Theorem 1.1. Let $(M, c)$ be a conformal manifold of dimension $n \geq 3$ and $\tilde{g}$ an ambient metric for $(M, c)$. If $x \in M$ and $z \in \mathcal{G}_{x}$, then

$$
\mathrm{ev}_{z}\left(\mathfrak{h o l} \mathfrak{l}_{x}\right)=\widetilde{\mathfrak{h o l}}_{z}
$$

An immediate corollary is the equality of restricted tractor and ambient holonomy groups in the odd-dimensional real-analytic case. Recall that if $(\mathcal{V}, \nabla)$ is a vector bundle with connection on a smooth manifold $M$ and $x \in M$, then the restricted holonomy group is

$$
\operatorname{Hol}_{x}^{0}(\mathcal{V}, \nabla)=\left\{L_{\gamma}\right\} \subset \operatorname{Aut} \mathcal{V}_{x}
$$


where $\gamma$ is a smooth contractible loop based at $x$ and $L_{\gamma}$ is the linear transformation of $\mathcal{V}_{x}$ obtained by parallel translation around $\gamma$. Just as with infinitesimal holonomy, for the tractor connection of a conformal manifold we have that if $z \in \mathcal{G}_{x}$, then $\operatorname{ev}_{z}\left(\operatorname{Hol}_{x}^{0}(\mathcal{T}, \nabla)\right)$ is an isomorphic copy of $\operatorname{Hol}_{x}^{0}(\mathcal{T}, \nabla)$ in $\operatorname{Aut} T_{z}(\mathcal{G} \times \mathbb{R})$.

Corollary 1.2. Let $(M, c)$ be an odd-dimensional real-analytic conformal manifold and $\tilde{g}$ a real-analytic ambient metric for $(M, c)$. If $x \in M$ and $z \in \mathcal{G}_{x}$, then

$$
\operatorname{ev}_{z}\left(\operatorname{Hol}_{x}^{0}(\mathcal{T}, \nabla)\right)=\operatorname{Hol}_{z}^{0}(T \widetilde{\mathcal{G}}, \widetilde{\nabla})
$$

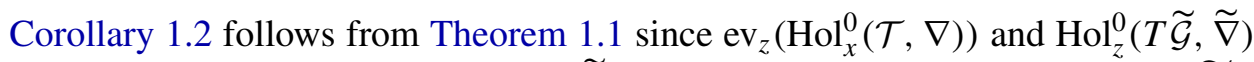
are connected Lie subgroups of Aut $T_{z} \widetilde{\mathcal{G}}$ with the same Lie algebra $\mathrm{ev}_{z}\left(\mathfrak{h o l}_{x}\right)=\widetilde{\mathfrak{h o l}}_{z}$.

The tractor bundle $\mathcal{T}$ carries a tractor metric $h$ of signature $(p+1, q+1)$ which is parallel with respect to $\nabla$. So by choosing a frame for $\mathcal{T}_{x}$, one can identify $\operatorname{Hol}^{0}(\mathcal{T}, \nabla)$ with a subgroup of $\operatorname{SO}_{e}(p+1, q+1)$ which is well-defined up to conjugacy independently of $x$ and the choice of frame (assuming $M$ is connected). Corollary 1.2 immediately implies:

Corollary 1.3. Let $(M, c)$ be an odd-dimensional connected real-analytic conformal manifold. Then its restricted conformal holonomy group $\operatorname{Hol}^{0}(\mathcal{T}, \nabla) \subset$ $\mathrm{SO}_{e}(p+1, q+1)$ is realizable as the restricted holonomy group of a real-analytic pseudo-Riemannian manifold of signature $(p+1, q+1)$.

Corollary 1.3 is interesting because of the wealth of known information concerning pseudo-Riemannian holonomy (in particular, Berger's list) and the restriction it places on conformal holonomy groups.

If a pseudo-Riemannian manifold admits a nonzero parallel tensor field, then its holonomy group is constrained to lie in the isotropy group consisting of the linear transformations preserving the tensor at a point. Of course, many interesting pseudo-Riemannian holonomy groups arise in this fashion. Likewise, interesting classes of conformal manifolds are characterized by admitting a parallel tractortensor field (i.e., a section of $\otimes^{r} \mathcal{T}^{*}$ for some $r \geq 1$ ) of a particular algebraic type. A precursor to Theorem 1.1 is the result of [Graham and Willse 2012] asserting that a parallel tractor-tensor field on a conformal manifold admits an extension to the ambient space which is parallel with respect to the ambient metric (to infinite order for $n$ odd, to order $\frac{n}{2}-1$ for $n$ even). This result was one motivation for our consideration of the question of equality of infinitesimal holonomy in general.

In order to prove Theorem 1.1, one must express the ambient connection and its curvature in tractor terms. Čap and Gover [2003] showed how the tractor bundle and connection could be written in ambient terms. This gives the inclusion $\mathrm{ev}_{z}\left(\mathfrak{h o l}_{x}\right) \subset \widetilde{\mathfrak{h o l}}_{z}$ in Theorem 1.1. Gover and Peterson [2003] reversed the direction and showed how to express the full ambient curvature and its covariant derivatives 
in terms of tractor calculus. Our proof of the reverse inclusion in Theorem 1.1, i.e., of ambient holonomy in tractor holonomy, is based on these relations.

In Section 2 we review the ambient metric construction and the realization of the tractor bundle and connection in ambient terms. In Section 3 we discuss infinitesimal holonomy and prove Theorem 1.1, in the process recalling the tractor expressions for the ambient curvature and connection.

\section{Ambient metrics and tractors}

We begin by reviewing background material concerning ambient metrics and tractors. The main reference for the material on ambient metrics is [Fefferman and Graham 2012]. References for the ambient formulation of tractors are [Čap and Gover 2003] and [Gover and Peterson 2003].

Let $(M, c)$ be a conformal manifold of dimension $n \geq 3$ and signature $(p, q)$, $p+q=n$. Metrics in the conformal class $c$ are sections of the metric bundle $\mathcal{G}:=\left\{\left(x, g_{x}\right): x \in M, g \in c\right\} \subset S^{2} T^{*} M$. Let $\pi: \mathcal{G} \rightarrow M$ denote the projection and $\delta_{s}: \mathcal{G} \rightarrow \mathcal{G}$ the dilations defined by $\delta_{s}\left(x, g_{x}\right)=\left(x, s^{2} g_{x}\right), s>0$. Let $T=\left.\frac{d}{d s} \delta_{s}\right|_{s=1}$ be the infinitesimal generator of the dilations. There is a tautological symmetric 2-tensor $\boldsymbol{g}$ on $\mathcal{G}$ defined for $X, Y \in T_{\left(x, g_{x}\right)} \mathcal{G}$ by $\boldsymbol{g}(X, Y)=g_{x}\left(\pi_{*} X, \pi_{*} Y\right)$.

Regard $\mathcal{G}$ as a hypersurface in $\mathcal{G} \times \mathbb{R}$ via $\iota(z)=(z, 0), z \in \mathcal{G}$. The variable in the $\mathbb{R}$ factor is denoted $\rho$. A straight preambient metric for $(M, c)$ is a smooth metric $\tilde{g}$ of signature $(p+1, q+1)$ on a dilation-invariant neighborhood $\widetilde{\mathcal{G}}$ of $\mathcal{G}$ satisfying

(1) $\delta_{s}^{*} \tilde{g}=s^{2} \tilde{g}$ for $s>0$;

(2) $\iota^{*} \tilde{g}=\boldsymbol{g}$;

(3) $\widetilde{\nabla} T=$ Id, where Id denotes the identity endomorphism and $\widetilde{\nabla}$ the Levi-Civita connection of $\tilde{g}$.

If $n$ is odd, an ambient metric for $(M, c)$ is a straight preambient metric for $(M, c)$ such that $\operatorname{Ric}(\tilde{g})$ vanishes to infinite order on $\mathcal{G}$. (To infinite order, the straightness condition (3) is a consequence of the infinite order vanishing of $\operatorname{Ric}(\tilde{g})$. But this is a nontrivial result (see [Fefferman and Graham 2012]), and it is convenient to have (3) holding in a full neighborhood of $\mathcal{G}$. So (3) is included in the definition.) There exists an ambient metric for $(M, c)$ and it is unique to infinite order up to pullback by a diffeomorphism defined on a dilation-invariant neighborhood of $\mathcal{G} \times \mathbb{R}$ which commutes with dilations and which restricts to the identity on $\mathcal{G}$. If $M$ is a real-analytic manifold and there is a real-analytic metric in the conformal class, then there exists a real-analytic ambient metric for $(M, c)$ satisfying $\operatorname{Ric}(\tilde{g})=0$ on some dilation-invariant $\widetilde{\mathcal{G}}$ as above.

In order to formulate the definition of ambient metrics for $n$ even, let $S_{I J}$ be a symmetric 2 -tensor field on an open neighborhood of $\mathcal{G}$ in $\mathcal{G} \times \mathbb{R}$ and $m \geq 0$. We write 
$S_{I J}=O_{I J}^{+}\left(\rho^{m}\right)$ if $S_{I J}=O\left(\rho^{m}\right)$ and, for each point $z \in \mathcal{G}$, the symmetric 2-tensor $\left(\iota^{*}\left(\rho^{-m} S\right)\right)(z)$ is of the form $\pi^{*} s$ for some symmetric 2-tensor $s$ at $x=\pi(z) \in M$ satisfying $\operatorname{tr}_{g_{x}} s=0$. If $n$ is even, an ambient metric for $(M, c)$ is a straight preambient metric such that $\operatorname{Ric}(\tilde{g})=O_{I J}^{+}\left(\rho^{n / 2-1}\right)$. There exists an ambient metric for $(M, c)$ and it is unique up to addition of a term which is $O_{I J}^{+}\left(\rho^{n / 2}\right)$ and up to pullback by a diffeomorphism defined on a dilation-invariant neighborhood of $\mathcal{G}$ which commutes with dilations and which restricts to the identity on $\mathcal{G}$. For $n$ even, a conformally invariant tensor, the ambient obstruction tensor, obstructs the existence of smooth solutions to $\operatorname{Ric}(\tilde{g})=O\left(\rho^{n / 2}\right)$.

Let $(M, c)$ be a conformal manifold with metric bundle $\mathcal{G} \underset{\rightarrow}{\rightarrow} M$. For $x \in M$, write $\mathcal{G}_{x}=\pi^{-1}(\{x\})$ for the fiber of $\mathcal{G}$ over $x$. Recall that the bundle $\mathcal{D}(w)$ of conformal densities of weight $w \in \mathbb{C}$ has fiber $\mathcal{D}_{x}(w)=\left\{f: \mathcal{G}_{x} \rightarrow \mathbb{C}:\left(\delta_{s}\right)^{*} f=s^{w} f, s>0\right\}$. Thus sections of $\mathcal{D}(w)$ on $M$ are functions on $\mathcal{G}$ homogeneous of degree $w$.

The standard tractor bundle and its normal connection can be similarly realized in terms of homogeneous vector fields on $\mathcal{G}_{x}$. As described in the introduction, the standard tractor bundle can be realized as the rank- $(n+2)$ vector bundle $\mathcal{T} \rightarrow M$ with fiber over $x$ given by (1-1). It can equivalently be described as an $\mathbb{R}_{+}$-quotient of $\left.T \widetilde{\mathcal{G}}\right|_{\mathcal{G}}$; see [Čap and Gover 2003]. If $\tilde{g}$ is an ambient metric for $(M, c)$ and if $U, W \in \mathcal{T}_{x}$, then $\tilde{g}(U, W)$ is homogeneous of degree 0 on $\mathcal{G}_{x}$, i.e., $\tilde{g}(U, W) \in \mathbb{R}$. Therefore $h(U, W)=\tilde{g}(U, W)$ defines a metric $h$ of signature $(p+1, q+1)$ on $\mathcal{T}$, the tractor metric. Since $T$ is homogeneous of degree 0 with respect to the $\delta_{s}$, it defines a section of $\mathcal{T}(1)$, where in general we denote the effect of tensoring a bundle with $\mathcal{D}(w)$ by appending $(w)$. The set of $U$ in (1-1) which at each point of $\mathcal{G}_{x}$ is a multiple of $T$ determines a subbundle of $\mathcal{T}$ which we denote $\operatorname{span}\{T\}$. Its orthogonal complement $\operatorname{span}\{T\}^{\perp}$ is the set of $U$ which at each point of $\mathcal{G}_{x}$ is tangent to $\mathcal{G}$. This gives the filtration

$$
0 \subset \operatorname{span}\{T\} \subset \operatorname{span}\{T\}^{\perp} \subset \mathcal{T}
$$

In order to realize the tractor connection, observe that $\pi_{*}: T \mathcal{G} \rightarrow T M$ induces a realization of the tangent bundle $T M$ as

$$
T_{x} M=\left\{\bar{\eta} \in \Gamma\left(\left.T \mathcal{G}\right|_{\mathcal{G}_{x}}\right):\left(\delta_{s}\right)^{*} \bar{\eta}=\bar{\eta}, s>0\right\} / \operatorname{span}\{T\},
$$

where here $\operatorname{span}\{T\}$ really means the constant multiples of $T$. If $\eta \in T_{x} M$, choose $\bar{\eta} \in \Gamma\left(\left.T \mathcal{G}\right|_{\mathcal{G}_{x}}\right)$ representing $\eta$. We will call such an $\bar{\eta}$ an invariant lift of $\eta$. Let $\tilde{g}$ be an ambient metric for $(M, c)$ and $\widetilde{\nabla}$ its Levi-Civita connection. If $U$ is a section of $\mathcal{T}$ near $x$, then $\widetilde{\nabla}_{\bar{\eta}} U \in \Gamma\left(\left.T \widetilde{\mathcal{G}}\right|_{\mathcal{G}_{x}}\right)$ makes sense since $U$ is defined on $\mathcal{G}$ and $\bar{\eta}$ is tangent to $\mathcal{G}$. The straightness of $\tilde{g}$ and the homogeneity of $U$ imply that $\widetilde{\nabla}_{T} U=0$. Therefore $\widetilde{\nabla}_{\bar{\eta}} U$ is independent of the choice of invariant lift $\bar{\eta}$. Also $\widetilde{\nabla}_{\bar{\eta}} U$ has the same homogeneity as $U$, so $\widetilde{\nabla}_{\bar{\eta}} U$ defines an element of $\mathcal{T}_{x}$. This realizes the tractor 
connection $\nabla$ on $\mathcal{T}$ :

$$
\nabla_{\eta} U=\widetilde{\nabla}_{\bar{\eta}} U
$$

The tractor metric $h$ is parallel with respect to $\nabla$ since $\widetilde{\nabla} \tilde{g}=0$. These realizations of the tractor metric and connection depend on the choice of ambient metric $\tilde{g}$. But the realizations obtained by changing $\tilde{g}$ by a diffeomorphism are equivalent.

The realization (1-1) of the tractor bundle induces the realizations

$$
\left(\otimes^{r} \mathcal{T}^{*}\right)_{x}=\left\{\chi \in \Gamma\left(\left.\otimes^{r} T^{*} \widetilde{\mathcal{G}}\right|_{\mathcal{G}_{x}}\right):\left(\delta_{s}\right)^{*} \chi=s^{r} \chi, s>0\right\}, \quad r \in \mathbb{N},
$$

of the bundles of cotractor-tensors, as well as the realization (1-3) of the bundle of tractor endomorphisms. The induced tractor connections on these bundles are also given in terms of the ambient connection and an invariant lift $\bar{\eta}$ as in (2-3). Throughout this paper we will identify weighted tractor-tensors with homogeneous sections of bundles on $\mathcal{G}$ as in (1-1), (1-3), (2-4).

The curvature $R$ of the tractor connection can be expressed in terms of the curvature $\widetilde{R}$ of an ambient metric. We have $R: \Lambda^{2} T M \rightarrow$ End $\mathcal{T}$ and $\widetilde{R}: \Lambda^{2} T \widetilde{\mathcal{G}} \rightarrow$ End $T \widetilde{\mathcal{G}}$. The straightness of the ambient metric implies that $T\lrcorner \widetilde{R}=0$ on $\mathcal{G}$. So if $\eta_{1}, \eta_{2} \in T_{x} M$ and $\bar{\eta}_{1}, \bar{\eta}_{2} \in \Gamma\left(\left.T \mathcal{G}\right|_{\mathcal{G}_{x}}\right)$ are invariant lifts, then $\widetilde{R}\left(\bar{\eta}_{1}, \bar{\eta}_{2}\right) \in \Gamma\left(\right.$ End $\left.\left.T \widetilde{\mathcal{G}}\right|_{\mathcal{G}_{x}}\right)$ is independent of the choices of $\bar{\eta}_{1}, \bar{\eta}_{2}$. Moreover, $\widetilde{R}\left(\bar{\eta}_{1}, \bar{\eta}_{2}\right)$ is homogeneous of degree 0 with respect to the $\delta_{s}$, so it realizes an element of End $\mathcal{T}_{x}$, and one has

$$
R\left(\eta_{1}, \eta_{2}\right)=\widetilde{R}\left(\bar{\eta}_{1}, \bar{\eta}_{2}\right) \text {. }
$$

We follow usual notational conventions. We label tensors on the ambient space and therefore also tractors with capital Latin indices and vectors on $M$ with lower case Latin indices. We use $\mathcal{E}$ to denote the space of smooth sections of a bundle on $M$, the bundle specified by the accompanying indices. Just as with the bundles themselves, we denote the spaces of sections of the corresponding weighted bundles by appending $(w)$. The notation $\mathcal{E}^{\Phi}(w)$ signifies the space of sections of a generic weighted tractor bundle, where $\Phi$ denotes an arbitrary collection of upper and lower capital indices. If $\Phi$ consists of $r$ upper indices and $s$ lower indices, we denote by $\widetilde{\mathcal{E}}^{\Phi}(w)$ the space of sections of $\left(\otimes^{r} T \widetilde{\mathcal{G}}\right) \otimes\left(\otimes^{s} T^{*} \widetilde{\mathcal{G}}\right)$ on $\widetilde{\mathcal{G}}$ of the same homogeneity degree as sections of $\mathcal{E}^{\Phi}(w)$, i.e., of homogeneity degree $w-r+s$. Ambient/tractor indices are raised and lowered using the ambient/tractor metric $\tilde{g}_{A B} / h_{A B}$ and lower case indices using the conformal metric $\boldsymbol{g}_{i j} \in \mathcal{E}_{i j}(2)$.

A choice of metric $g$ in the conformal class induces a splitting of the cotractor bundle

$$
\mathcal{T}^{*}=\mathcal{D}(-1) \oplus T^{*} M(1) \oplus \mathcal{D}(1) .
$$

This is the formulation in the original definition of the tractor bundle in [Bailey et al. 1994]. It can also be viewed in terms of the ambient realization by putting $\tilde{g}$ 
in normal form relative to $g$ (see [Gover and Peterson 2003] or [Graham and Willse 2012]). The three inclusions determined by this splitting determine sections

$$
X_{A} \in \mathcal{E}_{A}(1), \quad Z_{A}{ }^{i} \in \mathcal{E}_{A}{ }^{i}(-1), \quad Y_{A} \in \mathcal{E}_{A}(-1)
$$

so that

$$
V_{A}=\varphi X_{A}+\psi_{i} Z_{A}^{i}+\rho Y_{A}
$$

corresponds to $V_{A}=\left(\varphi, \psi_{i}, \rho\right) \in \mathcal{E}(-1) \oplus \mathcal{E}_{i}(1) \oplus \mathcal{E}(1)$. The sections $Y_{A}$ and $Z_{A}{ }^{i}$ are scale-dependent, i.e., they depend on the choice of $g$, while $X_{A}$ is scale-independent: $X^{A} \in \mathcal{E}^{A}(1)$ is another notation for the weighted tractor defined by the vector field $\left.T\right|_{\mathcal{G}}$.

\section{Holonomy}

Recall from the introduction that the infinitesimal holonomy $\mathfrak{h o l}_{x}$ of a vector bundle with connection $(\mathcal{V}, \nabla)$ on a manifold $M$ is defined pointwise by (1-2), and Theorem 1.1 is stated in terms of pointwise infinitesimal holonomy. The proof of Theorem 1.1 goes by induction on the order of differentiation. Thus it is natural to formulate an induction hypothesis involving objects which can be differentiated. So we introduce spaces consisting of global sections which restrict at each point to the infinitesimal holonomy. For $k \geq 2$, we define

$$
\text { (3-1) } \mathfrak{h o l}_{M}^{k}=\operatorname{span}_{C^{\infty}(M)}\left\{\nabla_{\eta_{l}} \nabla_{\eta_{l-1}} \ldots \nabla_{\eta_{3}}\left(R\left(\eta_{1}, \eta_{2}\right)\right): 2 \leq l \leq k, \eta_{1}, \ldots, \eta_{l} \in \mathfrak{X}(M)\right\}
$$

and

$$
\mathfrak{h o d} \mathfrak{l}_{M}=\bigcup_{k \geq 2} \mathfrak{h o r} \mathfrak{l}_{M}^{k}
$$

so that $\mathfrak{h o l} \mathfrak{l}_{M}^{k}, \mathfrak{h o l}{ }_{M} \subset \Gamma($ End $\mathcal{V})$. Clearly $\mathfrak{h o l}_{x}=\left\{E(x): E \in \mathfrak{h o l}{ }_{M}\right\}$. One has

$$
\left[\mathfrak{h o l} \mathfrak{l}_{M}^{k}, \mathfrak{h o l} \mathfrak{l}_{M}^{l}\right] \subset \mathfrak{h o l} \mathfrak{l}_{M}^{k+l} .
$$

In fact, the proof in [Kobayashi and Nomizu 1963] that $\mathfrak{h o l}_{x}$ is a subalgebra of End $\mathcal{V}_{x}$ establishes the analog of (3-2) in the principal bundle setting.

There is an alternate characterization of these spaces in terms of iterated covariant derivatives with respect to a coupled connection. If we choose arbitrarily a connection on $T M$ and denote also by $\nabla$ the coupled connection on $\mathcal{V} \otimes T M$, then the Leibniz formula and induction show that

$$
\text { (3-3) } \mathfrak{h o l}_{M}^{k}=\operatorname{span}_{C_{(M)}(M)}\left\{\left(\nabla^{l-2} R\right)\left(\eta_{1}, \eta_{2}, \ldots, \eta_{l}\right): 2 \leq l \leq k, \eta_{1}, \ldots, \eta_{l} \in \mathfrak{X}(M)\right\} .
$$

$R$ again denotes the curvature of the connection on $\mathcal{V}$. Here it is viewed as a section of $\Lambda^{2} T^{*} M \otimes$ End $\mathcal{V}$ and $\nabla^{l-2} R$ denotes its iterated covariant derivative with respect to the coupled connection. 
If $(M, c)$ is a conformal manifold, we take $\mathcal{V}=\mathcal{T}$ to be the tractor bundle with its normal connection and we denote the corresponding spaces by $\mathfrak{h o l}_{M}^{k}, \mathfrak{h o l}{ }_{M}$. As usual, via our realization (1-3) we identify elements of $\mathfrak{h o l}_{M}$ as global sections of End $\left.T(\mathcal{G} \times \mathbb{R})\right|_{\mathcal{G}}$ which are homogeneous of degree 0 with respect to the $\delta_{s}$.

For the ambient metric we modify the definition slightly to respect homogeneity. If $n$ is odd and $\tilde{g}$ is an ambient metric for $(M, c)$, we define for $k \geq 2$

$$
\widetilde{\mathfrak{h o l}}_{M}^{k}=\operatorname{span}_{C^{\infty}(M)}\left\{\left.\widetilde{\nabla}_{\tilde{\xi}_{l}} \widetilde{\nabla}_{\tilde{\xi}_{l-1}} \ldots \widetilde{\nabla}_{\tilde{\xi}_{3}}\left(\widetilde{R}\left(\tilde{\xi}_{1}, \tilde{\xi}_{2}\right)\right)\right|_{\mathcal{G}}: 2 \leq l \leq k, \tilde{\xi}_{1}, \ldots, \tilde{\xi}_{l} \in \mathfrak{X}_{0}(\widetilde{\mathcal{G}})\right\}
$$

where $\mathfrak{X}_{0}(\widetilde{\mathcal{G}})$ denotes the space of smooth vector fields on $\widetilde{\mathcal{G}}$ which are homogeneous of degree 0 with respect to the $\delta_{s}$ and $C^{\infty}(M)$ is viewed as the subspace of $C^{\infty}(\mathcal{G})$ of functions homogeneous of degree 0 . Observe that by definition, $\widetilde{\mathfrak{h o l}}_{M}^{k} \subset \Gamma\left(\right.$ End $\left.\left.T \widetilde{\mathcal{G}}\right|_{\mathcal{G}}\right)$ consists of sections which are homogeneous of degree 0 . If $n$ is even, we again define $\widetilde{\mathfrak{h o l}}_{M}^{k}$ for $k \geq 2$ by (3-4), except that we require that at most $\frac{n}{2}-2$ of the $\tilde{\xi}_{i}$ are somewhere transverse to $\mathcal{G}$. For general $n$, we then set

$$
\widetilde{\mathfrak{h o l}}_{M}=\bigcup_{k \geq 2} \widetilde{\mathfrak{h o l}}_{M}^{k}
$$

As above, $\widetilde{\mathfrak{h o l}}_{M}$ also has a description in terms of iterated derivatives of curvature:

$$
\widetilde{\mathfrak{h o l}}_{M}^{k}=\operatorname{span}_{C^{\infty}(M)}\left\{\left.\left(\widetilde{\nabla}^{l-2} \widetilde{R}\right)\left(\tilde{\xi}_{1}, \tilde{\xi}_{2}, \ldots, \tilde{\xi}_{l}\right)\right|_{\mathcal{G}}: 2 \leq l \leq k, \tilde{\xi}_{1}, \ldots, \tilde{\xi}_{l} \in \mathfrak{X}_{0}(\widetilde{\mathcal{G}})\right\}
$$

Here we take the coupling connection on $T \widetilde{\mathcal{G}}$ also to be the Levi-Civita connection $\widetilde{\nabla}$. As usual, for $n$ even we require that at most $\frac{n}{2}-2$ of the $\tilde{\xi}_{i}$ are somewhere transverse to $\mathcal{G}$. In this case, the equivalence of the descriptions (3-4) and (3-5) only holds for $k \leq \frac{n}{2}-1$, since $\widetilde{\nabla}_{\tilde{\xi}} \tilde{\eta}$ can be transverse to $\mathcal{G}$ when both $\left.\tilde{\xi}\right|_{\mathcal{G}}$ and $\left.\tilde{\eta}\right|_{\mathcal{G}}$ are tangent to $\mathcal{G}$.

We claim $\widetilde{\mathfrak{h o l}}_{z}=\left\{E(z): E \in \widetilde{\mathfrak{h o l}}_{M}\right\}$. To see this, choose a frame $\tilde{\zeta}_{0}, \tilde{\zeta}_{1}, \ldots, \tilde{\zeta}_{n+1}$ for $T \widetilde{\mathcal{G}}$ near $z$ such that $\left.\tilde{\zeta}_{A}\right|_{\mathcal{G}}$ is tangent to $\mathcal{G}$ for $1 \leq A \leq n+1$, and such that each $\tilde{\zeta}_{A}$ is homogeneous of degree 0 with respect to the $\delta_{s}$. By writing each $\tilde{\xi}_{i}$ in (1-4) as a linear combination of the $\tilde{\zeta}_{A}$, it is not hard to see that

$$
\widetilde{\mathfrak{h o l}}_{z}=\operatorname{span}_{\mathbb{R}}\left\{\widetilde{\nabla}_{\tilde{\zeta}_{A_{k}}} \widetilde{\nabla}_{\tilde{\zeta}_{A_{k-1}}} \ldots \widetilde{\nabla}_{\tilde{\zeta}_{A_{3}}}\left(\widetilde{R}\left(\tilde{\zeta}_{A_{1}}, \tilde{\zeta}_{A_{2}}\right)\right)(z): k \geq 2\right\},
$$

where for $n$ even at most $\frac{n}{2}-2$ of the indices $A_{1}, \ldots, A_{k}$ are equal to 0. It follows immediately that $\widetilde{\mathfrak{h o l}}_{z}=\left\{E(z): E \in \widetilde{\mathfrak{h o l}}_{M}\right\}$.

In light of these observations, it is clear that Theorem 1.1 is a consequence of the following theorem.

Theorem 3.1. Let $(M, c)$ be a conformal manifold of dimension $n \geq 3$ and $\tilde{g}$ an ambient metric for $(M, c)$. Then

$$
\mathfrak{h o l}_{M}=\widetilde{\mathfrak{h o l}}_{M}
$$


The inclusion $\mathfrak{h o l}_{M} \subset \mathfrak{h o l}_{M}$ follows immediately from the ambient realizations of the tractor connection and curvature. If $\eta_{1}, \ldots, \eta_{k} \in \mathfrak{X}(M)$ and $\bar{\eta}_{1}, \ldots, \bar{\eta}_{k}$ are invariant lifts, then (2-3), (2-5) give

$$
\nabla_{\eta_{k}} \nabla_{\eta_{k-1}} \cdots \nabla_{\eta_{3}}\left(R\left(\eta_{1}, \eta_{2}\right)\right)=\widetilde{\nabla}_{\bar{\eta}_{k}} \widetilde{\nabla}_{\bar{\eta}_{k-1}} \ldots \widetilde{\nabla}_{\bar{\eta}_{3}}\left(\widetilde{R}\left(\bar{\eta}_{1}, \bar{\eta}_{2}\right)\right),
$$

so $\mathfrak{h o l}_{M} \subset \widetilde{\mathfrak{h o l}}_{M}$. The right-hand side is in $\widetilde{\mathfrak{h o l}}_{M}$ also for $n$ even since none of the $\bar{\eta}_{i}$ are transverse to $\mathcal{G}$.

We remark that (3-6) is already sufficient to prove Theorem 3.1, and therefore also Theorem 1.1, when $n=4$. In fact, when $n=4$, each $\left.\tilde{\xi}_{i}\right|_{\mathcal{G}}$ in (3-4) is required to be everywhere tangent to $\mathcal{G}$, so is an invariant lift of some $\eta_{i} \in \mathfrak{X}(M)$.

To prove the opposite inclusion $\widetilde{\mathfrak{h o l}}_{M} \subset \mathfrak{h o l}_{M}$, we must rewrite expressions of the form $\left.\widetilde{\nabla}_{\tilde{\xi}_{l}} \widetilde{\nabla}_{\tilde{\xi}_{l-1}} \ldots \widetilde{\nabla}_{\tilde{\xi}_{3}}\left(\widetilde{R}\left(\tilde{\xi}_{1}, \tilde{\xi}_{2}\right)\right)\right|_{\mathcal{G}}$ purely in tractor terms when the $\tilde{\xi}_{i}$ are allowed to be transverse to $\mathcal{G}$. We do this using tractor representations of the curvature and connection of the ambient metric derived in [Gover and Peterson 2003]. These representations are expressed in terms of the splitting (2-6), (2-7) of the cotractor bundle determined by a choice of metric $g \in c$. Consider first the case $n$ odd.

Proof of Theorem 3.1 for $n$ odd. We show by induction on $k \geq 2$ that $\widetilde{\mathfrak{h o l}}_{M}^{k} \subset \mathfrak{h o l}_{M}$. For $k=2$, we use the tractor expression for ambient curvature

$$
\left.\widetilde{R}_{A B}{ }^{P} Q\right|_{\mathcal{G}}=Z_{A}^{a} Z_{B}^{b} R_{a b}{ }^{P} Q-\frac{2}{n-4} X_{[A} Z_{B]}^{b} \nabla^{c} R_{c b}{ }^{P} Q .
$$

This is (13), (35) of [Gover and Peterson 2003]. The $\nabla^{c}$ on the right-hand side refers to the connection obtained by coupling the tractor connection with the Levi-

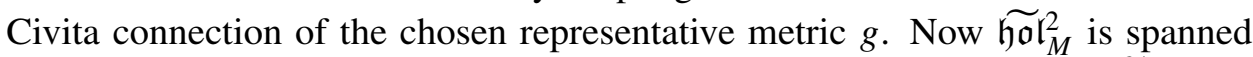
by contractions of the left-hand side against $\tilde{\xi}_{1}^{A} \tilde{\xi}_{2}^{B}$, where $\tilde{\xi}_{1}, \tilde{\xi}_{2} \in \mathfrak{X}_{0}(\widetilde{\mathcal{G}})$. It is evident that after such a contraction, the first term on the right-hand side is in $\mathfrak{h o l}_{M}^{2}$. For the second term, write $\nabla^{c} R_{c b}{ }^{P} Q=g^{c d} \nabla_{c} R_{d b}{ }^{P} Q$ and introduce a partition of unity subordinate to a covering of $M$ in each open set of which $\boldsymbol{g}^{c d}$ can be expressed as a smooth linear combination of tensor products of vector fields. It follows that after contraction with $\tilde{\xi}_{1}^{A} \tilde{\xi}_{2}^{B}$, the second term is in $\mathfrak{h o l}_{M}^{3}$. Thus the initial $k=2$ step of the induction is established.

The induction step for higher $k$ will be carried out using the tractor- $D$ operator. If $\Phi$ denotes an arbitrary collection of upper and/or lower tractor indices, then

$$
D_{A}: \mathcal{E}^{\Phi}(w) \rightarrow \mathcal{E}_{A}^{\Phi}(w-1)
$$

is defined in terms of the splitting determined by a representative metric $g$ by

$$
D_{A} V=w(n+2 w-2) Y_{A} V+(n+2 w-2) Z_{A}{ }^{a} \nabla_{a} V-X_{A} \square V,
$$


where $\square V=\nabla^{i} \nabla_{i} V+w J V$ and $J=R /(2(n-1)) . D_{A}$ can also be expressed in ambient terms:

$$
D_{A} V=\left.(n+2 w-2) \widetilde{\nabla}_{A} \widetilde{V}\right|_{\mathcal{G}}-\left.X_{A}(\widetilde{\Delta} \tilde{V})\right|_{\mathcal{G}} .
$$

These are (8), (31) of [Gover and Peterson 2003]. On the right-hand side, $\widetilde{V} \in \widetilde{\mathcal{E}}^{\Phi}(w)$ is an arbitrary homogeneous extension of $V \in \mathcal{E}^{\Phi}(w)$ and $\widetilde{\Delta}$ denotes the ambient Laplacian acting on the corresponding space of tensors: $\widetilde{\Delta}=\widetilde{\nabla}^{I} \widetilde{\nabla}_{I}$. The expression on the right-hand side turns out to be independent of the choice of $\widetilde{V}$.

Assume now that $k \geq 2$ and $\widetilde{\mathfrak{h o l}}_{M}^{k} \subset \mathfrak{h o l}_{M}$. According to (3-5), in order to prove

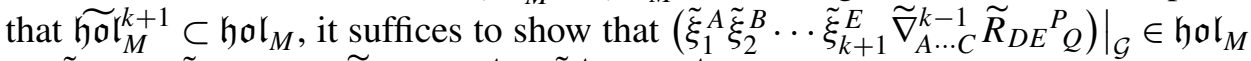
for $\tilde{\xi}_{1}, \ldots, \tilde{\xi}_{k+1} \in \mathfrak{X}_{0}(\widetilde{\mathcal{G}})$. Set $\xi_{s}^{A}=\left.\tilde{\xi}_{s}^{A}\right|_{\mathcal{G}} \in \mathcal{E}^{A}(1), 1 \leq s \leq k+1$.

Define

$$
\widetilde{V}=\widetilde{\nabla}_{B \cdots C}^{k-2} \widetilde{R}_{D E}^{P} Q \in \widetilde{\mathcal{E}}_{B \cdots E}^{P}{ }_{Q}(-k)
$$

and rewrite (3-8) as

$$
\left.(n-2 k-2) \widetilde{\nabla}_{A} \widetilde{V}\right|_{\mathcal{G}}=D_{A} V+\left.X_{A}(\tilde{\Delta} \widetilde{V})\right|_{\mathcal{G}},
$$

where $V:=\left.\widetilde{V}\right|_{\mathcal{G}} \in \mathcal{E}_{B \cdots E}{ }^{P}(-k)$. Since the coefficient $(n-2 k-2)$ is nonzero for $n$ odd, it suffices to show that

$$
\xi_{1}^{A} \cdots \xi_{k+1}^{E} D_{A} V_{B \cdots E}^{P} Q \in \mathfrak{h o l}_{M}
$$

and

$$
\left.\tilde{\xi}_{2}^{B} \cdots \tilde{\xi}_{k+1}^{E} \widetilde{\Delta} \widetilde{V}_{B \cdots E}{ }^{P}\right|_{\mathcal{G}} \in \mathfrak{h o l}_{M} .
$$

For (3-9), contract (3-7) against $\xi_{1}^{A} \cdots \xi_{k+1}^{E}$. The first term on the right-hand side gives a multiple of

$$
\left(\xi_{1}^{A} Y_{A}\right) \xi_{2}^{B} \cdots \xi_{k+1}^{E} V_{B \cdots E}{ }_{Q},
$$

which is in $\mathfrak{h o l}_{M}$ by the induction hypothesis. The second term on the right-hand side gives a multiple of

$$
\left(\xi_{1}^{A} Z_{A}{ }^{a}\right) \xi_{2}^{B} \cdots \xi_{k+1}^{E} \nabla_{a} V_{B \cdots E}^{P}{ }_{Q} .
$$

If we set $\eta^{a}=\xi_{1}^{A} Z_{A}{ }^{a}$, then this can be rewritten as

$$
\begin{aligned}
& \eta^{a} \xi_{2}^{B} \cdots \xi_{k+1}^{E} \nabla_{a} V_{B \cdots E}{ }^{P} Q \\
& =\nabla_{\eta}\left(\xi_{2}^{B} \cdots \xi_{k+1}^{E} V_{B \cdots E}{ }^{P} Q\right)-\sum_{s=2}^{k+1} \xi_{2}^{B} \cdots\left(\nabla_{\eta} \xi_{s}^{R}\right) \cdots \xi_{k+1}^{E} V_{B \cdots R} \cdots E^{P} .
\end{aligned}
$$

The induction hypothesis shows that $\xi_{2}^{B} \cdots \xi_{k+1}^{E} V_{B \cdots E}{ }_{Q} \in \mathfrak{h o l}_{M}$, so we conclude that $\nabla_{\eta}\left(\xi_{2}^{B} \cdots \xi_{k+1}^{E} V_{B \cdots E} P_{Q}\right) \in \mathfrak{h o l}_{M}$. Each term in the sum on the right-hand side is clearly in $\mathfrak{h o l}{ }_{M}$ by the induction hypothesis. Thus the contraction of the second 
term of the right-hand side of (3-7) is in $\mathfrak{h o l}_{M}$. The third term of (3-7) is handled similarly, namely by expanding the difference

$$
\xi_{2}^{B} \cdots \xi_{k+1}^{E} \nabla^{c} \nabla_{c} V_{B \cdots E}{ }^{P} Q-\nabla^{c} \nabla_{c}\left(\xi_{2}^{B} \cdots \xi_{k+1}^{E} V_{B \cdots E}{ }^{P} Q\right)
$$

using the Leibniz rule and introducing a partition of unity to rewrite sections of tensor product bundles as sums of tensor products of sections of the factors as in the proof in the case $k=2$. This concludes the proof of (3-9).

It remains to prove (3-10). Now $\widetilde{\Delta} \widetilde{V}=\widetilde{\Delta} \widetilde{\nabla}^{k-2} \widetilde{R}$. It is well-known that the Laplacian of an iterated covariant derivative of the curvature tensor of a Ricci-flat metric can be reexpressed as a linear combination of quadratic terms in curvature by commuting both derivatives in $\widetilde{\Delta}$ all the way to the right and applying the second Bianchi identity. We will argue using the induction hypothesis that each commutator term is already in $\mathfrak{h o l}{ }_{M}$.

Write

$$
\widetilde{\Delta} \widetilde{\nabla}^{k-2} \widetilde{R}_{D E}^{P} Q=\tilde{g}^{I J} \widetilde{\nabla}_{I} \widetilde{\nabla}_{J} \widetilde{\nabla}^{k-2} \widetilde{R}_{D E}^{P} Q .
$$

First commute $\widetilde{\nabla}_{J}$ to the right of all derivatives in $\widetilde{\nabla}^{k-2}$. Modulo commutator terms, one obtains

$$
\tilde{g}^{I J} \widetilde{\nabla}_{I} \widetilde{\nabla}^{k-2} \widetilde{\nabla}_{J} \widetilde{R}_{D E}^{P} Q=\tilde{g}^{I J} \widetilde{\nabla}_{I} \widetilde{\nabla}^{k-2} \widetilde{\nabla}_{D} \widetilde{R}_{J E}^{P}{ }^{P}+\tilde{g}^{I J} \widetilde{\nabla}_{I} \widetilde{\nabla}^{k-2} \widetilde{\nabla}_{E} \widetilde{R}_{D J}^{P} Q .
$$

Now commuting $\widetilde{\nabla}_{I}$ all the way to the right shows that modulo commutators the above is equal to

$$
\tilde{g}^{I J} \widetilde{\nabla}^{k-2} \widetilde{\nabla}_{D} \widetilde{\nabla}_{I} \widetilde{R}_{J E}^{P} Q+\tilde{g}^{I J} \widetilde{\nabla}^{k-2} \widetilde{\nabla}_{E} \widetilde{\nabla}_{I} \widetilde{R}_{D J}^{P}{ }_{Q} .
$$

This vanishes on $\mathcal{G}$ by the second Bianchi identity and the infinite-order vanishing of Ric $\tilde{g}$.

To analyze the commutator terms, it is convenient to suppress writing the End $T \widetilde{\mathcal{G}}$ indices ${ }_{Q}$. We will denote by $\widetilde{\mathcal{R}}_{B C}$ the curvature tensor of $\tilde{g}$ viewed as an End $T \widetilde{\mathcal{G}}$ valued section of $\Lambda^{2} T^{*} \widetilde{\mathcal{G}}$. If $U$ is an End $T \widetilde{\mathcal{G}}$-valued section of $\otimes^{r} T^{*} \widetilde{\mathcal{G}}$ and $V$ is an End $T \widetilde{\mathcal{G}}$-valued section of $\otimes^{s} T^{*} \widetilde{\mathcal{G}}$, we will denote by $[U, V]$ the End $T \widetilde{\mathcal{G}}$-valued section of $\otimes^{r+s} T^{*} \widetilde{\mathcal{G}}$ which is the commutator in the End $T \widetilde{\mathcal{G}}$ indices and the tensor product in the $T^{*} \widetilde{\mathcal{G}}$ indices. The Leibniz formula gives

$$
\widetilde{\nabla}[U, V]=[\widetilde{\nabla} U, V]+[U, \widetilde{\nabla} V] .
$$

The Ricci identity for commuting covariant derivatives can be written

$$
\left[\widetilde{\nabla}_{B}, \widetilde{\nabla}_{C}\right] U=\widetilde{\mathcal{R}}_{B C} \cdot U+\left[\widetilde{\mathcal{R}}_{B C}, U\right],
$$

where $\widetilde{\mathcal{R}}_{B C} . U$ denotes the action of the endomorphism $\widetilde{\mathcal{R}}_{B C}$ on the $\otimes^{r} T^{*} \widetilde{\mathcal{G}}$ indices of $U$. 
Every commutator which arose in the above argument was of the form

$$
\widetilde{\nabla}^{i}\left[\widetilde{\nabla}_{B}, \widetilde{\nabla}_{C}\right] \widetilde{\nabla}^{j} \widetilde{\mathcal{R}}
$$

for some choice of indices $B, C$, where $i \geq 0, j \geq 0$, and $i+j=k-2$. Express the commutator $\left[\widetilde{\nabla}_{B}, \widetilde{\nabla}_{C}\right] \widetilde{\nabla}^{j} \widetilde{\mathcal{R}}$ using (3-12) with $U=\widetilde{\nabla}^{j} \widetilde{\mathcal{R}}$. The first term on the right-hand side of (3-12) gives terms of the form $\widetilde{\nabla}^{i}\left(\widetilde{\mathcal{R}} . \widetilde{\nabla}^{j} \widetilde{\mathcal{R}}\right)$. Expanding the $\widetilde{\nabla}^{i}$ with the Leibniz rule, it is clear that one obtains a sum of terms, each of which has the form

$$
\operatorname{contr}\left(\widetilde{\nabla} p \widetilde{\mathcal{R}} \otimes \widetilde{\nabla}^{q} \widetilde{\mathcal{R}}\right),
$$

with $p \geq 0, q \geq 0$, and $p+q=k-2$. Here contr indicates a single contraction of the upper End $T^{*} \widetilde{\mathcal{G}}$ index of $\widetilde{\nabla}^{p} \widetilde{R}$ against one of the $\otimes^{q+2} T^{*} \widetilde{\mathcal{G}}$ indices of $\widetilde{\nabla} q \widetilde{\mathcal{R}}$. In particular, the suppressed End $T^{*} \widetilde{\mathcal{G}}$ indices are those on $\widetilde{\nabla} q \widetilde{\mathcal{R}}$. The second term on the right-hand side of (3-12) gives terms of the form $\widetilde{\nabla}^{i}\left[\widetilde{\mathcal{R}}, \widetilde{\nabla^{j}} \widetilde{\mathcal{R}}\right]$. Expanding the $\widetilde{\nabla}^{i}$ using (3-11), one obtains a sum of terms of the form

$$
\left[\widetilde{\nabla}^{p} \widetilde{\mathcal{R}}, \widetilde{\nabla}^{q} \widetilde{\mathcal{R}}\right],
$$

again with $p \geq 0, q \geq 0$, and $p+q=k-2$.

We need to show (3-10). Suppressing the End $T \widetilde{\mathcal{G}}$ indices, we have

$$
\left.\tilde{\xi}_{2}^{B} \cdots \tilde{\xi}_{k+1}^{E} \widetilde{\Delta} \widetilde{V}_{B \cdots E}\right|_{\mathcal{G}}=\left.\tilde{\xi}_{2}^{B} \cdots \tilde{\xi}_{k+1}^{E} \tilde{g}^{I J} \widetilde{\nabla}_{I} \widetilde{\nabla}_{J} \widetilde{\nabla}_{B \cdots C}^{k-2} \widetilde{\mathcal{R}}_{D E}\right|_{\mathcal{G}} .
$$

Upon commuting $\widetilde{\nabla}_{I}$ and $\widetilde{\nabla}_{J}$ to the right as described above, it follows that this may be written as a sum of contractions of terms of the form (3-13), (3-14) against $\tilde{\xi}_{i}$ and $\tilde{g}^{I J}$ with all indices contracted except for the suppressed End $T \widetilde{\mathcal{G}}$ indices. In a term (3-13), the free End $T \widetilde{\mathcal{G}}$ indices are those on the second factor $\widetilde{\nabla}^{q} \widetilde{\mathcal{R}}$. Consequently, we can introduce a partition of unity and express locally the tensor arising from $\tilde{g}^{I J}, \widetilde{\nabla}^{p} \widetilde{\mathcal{R}}$, and the $\tilde{\xi}_{i}$ which contracts against the other $q+2$ indices of $\widetilde{\nabla} q \widetilde{\mathcal{R}}$ as a sum of tensor products of vector fields. Since $q \leq k-2$, it follows by the induction hypothesis that all these terms are in $\mathfrak{h o l}_{M}$ when restricted to $\mathcal{G}$. In a term (3-14), all the indices except the endomorphism indices are contracted against $\tilde{g}^{I J}$ and the $\tilde{\xi}_{i}$. Again use a partition of unity and express locally $\tilde{g}^{I J}$ as a sum of tensor products of vector fields. Then the induction hypothesis implies that the restriction to $\mathcal{G}$ of the contractions against $\widetilde{\nabla}^{p} \widetilde{\mathcal{R}}$ and $\widetilde{\nabla}^{q} \widetilde{\mathcal{R}}$ are separately in $\mathfrak{h o l}_{M}$. It follows from (3-2) that the commutator is also in $\mathfrak{h o l}_{M}$.

Proof of Theorem 3.1 for $n$ even. We have already observed that (3-6) is sufficient to prove the case $n=4$. So we assume that $n \geq 6$. We next observe that the same argument used for $n$ odd applies also when $n$ is even to show $\widetilde{h o l}_{M}^{n / 2-1} \subset \mathfrak{h o l}_{M}$. In fact, up to this order the relevant constant $n+2 w-2$ in (3-8) is nonzero and the argument only uses $\operatorname{Ric}(\tilde{g})=O\left(\rho^{n / 2-1}\right)$. 
For $n \geq 6$ even, we prove $\widetilde{\mathfrak{h o l}}_{M}^{k} \subset \mathfrak{h o l}_{M}$ by induction on $k$, beginning with the case $k=\frac{n}{2}-1$. So assume for some $k \geq \frac{n}{2}-1$ that $\widetilde{\mathfrak{h o l}}_{M}^{k} \subset \mathfrak{h o l}_{M}$ and we will show $\widetilde{\mathfrak{h o l}}_{M}^{k+1} \subset \mathfrak{h o l}_{M}$. According to (3-4), we have to show that

$$
\left.\widetilde{\nabla}_{\tilde{\xi}_{k+1}} \widetilde{\nabla}_{\tilde{\xi}_{k}} \ldots \widetilde{\nabla}_{\tilde{\xi}_{3}}\left(\widetilde{R}\left(\tilde{\xi}_{1}, \tilde{\xi}_{2}\right)\right)\right|_{\mathcal{G}} \in \mathfrak{h o l} \mathfrak{l}_{M}
$$

whenever $\tilde{\xi}_{1}, \ldots, \tilde{\xi}_{k+1} \in \mathfrak{X}_{0}(\widetilde{\mathcal{G}})$ and at most $\frac{n}{2}-2$ of the $\tilde{\xi}_{i}$ are somewhere transverse to $\mathcal{G}$. Since $k+1 \geq \frac{n}{2}$, at least two of the $\tilde{\xi}_{i}$ are everywhere tangent to $\mathcal{G}$. If $\tilde{\xi}_{k+1}$ is everywhere tangent to $\mathcal{G}$, then its restriction to $\mathcal{G}$ is the invariant lift of some $\eta \in \mathfrak{X}(M)$. In this case (2-3) gives

$$
\left.\widetilde{\nabla}_{\tilde{\xi}_{k+1}} \widetilde{\nabla}_{\tilde{\xi}_{k}} \ldots \widetilde{\nabla}_{\tilde{\xi}_{3}}\left(\widetilde{R}\left(\tilde{\xi}_{1}, \tilde{\xi}_{2}\right)\right)\right|_{\mathcal{G}}=\nabla_{\eta}\left(\left.\widetilde{\nabla}_{\tilde{\xi}_{k}} \ldots \widetilde{\nabla}_{\tilde{\xi}_{3}}\left(\widetilde{R}\left(\tilde{\xi}_{1}, \tilde{\xi}_{2}\right)\right)\right|_{\mathcal{G}}\right)
$$

The induction hypothesis shows that $\left.\widetilde{\nabla}_{\tilde{\xi}_{k}} \ldots \widetilde{\nabla}_{\tilde{\xi}_{3}}\left(\widetilde{R}\left(\tilde{\xi}_{1}, \tilde{\xi}_{2}\right)\right)\right|_{\mathcal{G}} \in \mathfrak{h o l} \mathfrak{l}_{M}$, from which it follows that $\nabla_{\eta}\left(\left.\widetilde{\nabla}_{\tilde{\xi}_{k}} \ldots \widetilde{\nabla}_{\tilde{\xi}_{3}}\left(\widetilde{R}\left(\tilde{\xi}_{1}, \tilde{\xi}_{2}\right)\right)\right|_{\mathcal{G}}\right) \in \mathfrak{h}_{\mathfrak{s} \mathfrak{l}_{M}}$, as desired.

If $\tilde{\xi}_{i}$ is everywhere tangent to $\mathcal{G}$ for some $i, 3 \leq i \leq k$, then we can commute $\widetilde{\nabla}_{\tilde{\xi}_{i}}$ all the way to the left and reduce to the previous case. Modulo relabeling the indices, each commutator is of the form

$$
\begin{aligned}
\widetilde{\nabla}_{\tilde{\xi}_{k+1}} \ldots \widetilde{\nabla}_{\tilde{\xi}_{j+1}}\left[\widetilde{\nabla}_{\tilde{\xi}_{j},}, \widetilde{\nabla}_{\left.\tilde{\xi}_{j-1}\right]}\right] \widetilde{\nabla}_{\tilde{\xi}_{j-2}} \ldots \widetilde{\nabla}_{\tilde{\xi}_{3}} \widetilde{R}_{\left(\tilde{\xi}_{1}, \tilde{\xi}_{2}\right)} \\
=\widetilde{\nabla}_{\tilde{\xi}_{k+1}} \ldots \widetilde{\nabla}_{\tilde{\xi}_{j+1}} \widetilde{\nabla}_{\left[\tilde{\xi}_{j}, \tilde{\xi}_{j-1}\right]} \widetilde{\nabla}_{\tilde{\xi}_{j-2}} \ldots \widetilde{\nabla}_{\tilde{\xi}_{3}} \widetilde{R}\left(\tilde{\xi}_{1}, \tilde{\xi}_{2}\right) \\
\quad+\widetilde{\nabla}_{\tilde{\xi}_{k+1}} \ldots \widetilde{\nabla}_{\tilde{\xi}_{j+1}}\left[\widetilde{R}\left(\tilde{\xi}_{j}, \tilde{\xi}_{j-1}\right), \widetilde{\nabla}_{\tilde{\xi}_{j-2}} \ldots \widetilde{\nabla}_{\tilde{\xi}_{3}} \widetilde{R}\left(\tilde{\xi}_{1}, \tilde{\xi}_{2}\right)\right] .
\end{aligned}
$$

In the first term on the right-hand side, the number of differentiations has decreased by 1 without increasing the number of vector fields somewhere transverse to $\mathcal{G}$, since the commutator of two vector fields tangent to $\mathcal{G}$ is also tangent to $\mathcal{G}$. So the restriction to $\mathcal{G}$ of this term is in $\mathfrak{h o l}_{M}$ by the induction hypothesis. In the second term on the right-hand side, expand the derivatives outside the commutator using the Leibniz rule. One obtains a linear combination of commutators of covariant derivatives of curvature endomorphisms. The restriction to $\mathcal{G}$ of each such covariant derivative itself is in $\mathfrak{h o l}_{M}$ by the induction hypothesis. Equation (3-2) then shows that the commutator is in $\mathfrak{h o l}{ }_{M}$.

Finally we must consider the possibility that none of $\tilde{\xi}_{3}, \ldots, \tilde{\xi}_{k+1}$ is everywhere tangent to $\mathcal{G}$. (This can only happen in the beginning case $k=\frac{n}{2}-1$, but we will not use this.) It must be that $\tilde{\xi}_{1}$ and $\tilde{\xi}_{2}$ are everywhere tangent to $\mathcal{G}$. In this case, we apply the second Bianchi identity to write

$$
\begin{aligned}
\widetilde{\nabla}_{\tilde{\xi}_{3}} \widetilde{R}\left(\tilde{\xi}_{1}, \tilde{\xi}_{2}\right)=\widetilde{\nabla}_{\tilde{\xi}_{1}} \widetilde{R}\left(\tilde{\xi}_{3}, \tilde{\xi}_{2}\right)+\widetilde{\nabla}_{\tilde{\xi}_{2}} \widetilde{R}\left(\tilde{\xi}_{1}, \tilde{\xi}_{3}\right)+\widetilde{R}\left(\widetilde{\nabla}_{\tilde{\xi}_{3}} \tilde{\xi}_{1}, \tilde{\xi}_{2}\right)+\widetilde{R}\left(\tilde{\xi}_{1}, \widetilde{\nabla}_{\tilde{\xi}_{3}} \tilde{\xi}_{2}\right) \\
-\widetilde{R}\left(\widetilde{\nabla}_{\tilde{\xi}_{1}} \tilde{\xi}_{3}, \tilde{\xi}_{2}\right)-\widetilde{R}\left(\tilde{\xi}_{1}, \widetilde{\nabla}_{\tilde{\xi}_{2}} \tilde{\xi}_{3}\right)+\widetilde{R}\left(\widetilde{\nabla}_{\tilde{\xi}_{1}} \tilde{\xi}_{2}-\widetilde{\nabla}_{\tilde{\xi}_{2}} \tilde{\xi}_{1}, \tilde{\xi}_{3}\right) .
\end{aligned}
$$

The first two terms of the right-hand side reduce to the previous case. The next four terms reduce to the induction hypothesis since $\tilde{\xi}_{1}$ and $\tilde{\xi}_{2}$ are tangential and at 
least one occurs as an argument in each term, so the number of transversal vector fields does not increase. The last term also reduces to the induction hypothesis since $\widetilde{\nabla}_{\tilde{\xi}_{1}} \tilde{\xi}_{2}-\widetilde{\nabla}_{\tilde{\xi}_{2}} \tilde{\xi}_{1}=\left[\tilde{\xi}_{1}, \tilde{\xi}_{2}\right]$ is tangential.

\section{References}

[Alt 2012] J. Alt, “Transitive conformal holonomy groups”, Cent. Eur. J. Math. 10:5 (2012), 17101720. MR 2949648 Zbl 1278.53045

[Armstrong 2007] S. Armstrong, "Definite signature conformal holonomy: a complete classification", J. Geom. Phys. 57:10 (2007), 2024-2048. MR 2348277 Zbl 05201910

[Armstrong and Leitner 2012] S. Armstrong and F. Leitner, "Decomposable conformal holonomy in Riemannian signature”, Math. Nachr. 285:2-3 (2012), 150-163. MR 2881272 Zbl 1246.53064

[Bailey et al. 1994] T. N. Bailey, M. G. Eastwood, and A. R. Gover, "Thomas's structure bundle for conformal, projective and related structures”, Rocky Mountain J. Math. 24:4 (1994), 1191-1217. MR 1322223 Zbl 0828.53012

[Čap and Gover 2003] A. Čap and A. R. Gover, "Standard tractors and the conformal ambient metric construction”, Ann. Global Anal. Geom. 24:3 (2003), 231-259. MR 1996768 Zbl 1039.53021

[Fefferman and Graham 1985] C. Fefferman and C. R. Graham, "Conformal invariants", pp. 95-116 in The mathematical heritage of Élie Cartan (Lyon, 1984), Astérisque (numéro hors série), Société Mathématique de France, Paris, 1985. MR 87g:53060 Zbl 0602.53007

[Fefferman and Graham 2012] C. Fefferman and C. R. Graham, The ambient metric, Annals of Mathematics Studies 178, Princeton University Press, 2012. MR 2858236 Zbl 1243.53004

[Gover and Peterson 2003] A. R. Gover and L. J. Peterson, "Conformally invariant powers of the Laplacian, $Q$-curvature, and tractor calculus”, Comm. Math. Phys. 235:2 (2003), 339-378. MR 1969732 Zbl 1022.58014

[Graham and Willse 2012] C. R. Graham and T. Willse, "Parallel tractor extension and ambient metrics of holonomy split $G_{2}$ ”, J. Differential Geom. 92:3 (2012), 463-505. MR 3005060 Zbl 1268.53075

[Kobayashi and Nomizu 1963] S. Kobayashi and K. Nomizu, Foundations of differential geometry, I, Interscience Publishers, New York, 1963. Zbl 0119.37502

[Leistner 2006] T. Leistner, "Conformal holonomy of C-spaces, Ricci-flat, and Lorentzian manifolds", Differential Geom. Appl. 24:5 (2006), 458-478. MR 2254049 Zbl 1109.53052

[Leitner 2005] F. Leitner, "Conformal Killing forms with normalisation condition”, pp. 279-292 in Proceedings of the 24th winter school "geometry and physics" (Srní, Czech Republic, 2005), II 75, 2005. MR 2152367 Zbl 1101.53040

[Lischewski 2015] A. Lischewski, "Reducible conformal holonomy in any metric signature and application to twistor spinors in low dimension", Differential Geom. Appl. 40 (2015), 252-268. MR 3333107 Zbl 1318.53047

[Nijenhuis 1953a] A. Nijenhuis, "On the holonomy groups of linear connections, IA: General properties of affine connections", Nederl. Akad. Wetensch. Proc. Ser. A. 15 (1953), 233-240. MR 0063737 Zbl 0051.13203

[Nijenhuis 1953b] A. Nijenhuis, "On the holonomy groups of linear connections, IB: General properties of affine connections", Nederl. Akad. Wetensch. Proc. Ser. A 15 (1953), 241-249. MR 0063737 Zbl 0051.13203

[Nijenhuis 1954] A. Nijenhuis, "On the holonomy groups of linear connections, II: Properties of general linear connections", Nederl. Akad. Wetensch. Proc. Ser. A. 16 (1954), 17-25. MR 0063738 Zbl 0055.40703 
Received April 3, 2015. Revised March 9, 2016.

ANDREAS ČAP

FACULTY OF MATHEMATICS

UNIVERSITY OF VIENNA

OSKAR-MORGENSTERN-PLATZ 1

1090 WIEN

AUSTRIA

andreas.cap@univie.ac.at

A. ROD GOVER

DEPARTMENT OF MATHEMATICS

THE UNIVERSITY OF AUCKLAND

PRIVATE BAG 92019

AUCKLAND 1142

NEW ZEALAND

r.gover@auckland.ac.nz

C. ROBIN GRAHAM

DEPARTMENT OF MATHEMATICS

UNIVERSITY OF WASHINGTON

BOX 354350

SEATTLE, WA 98195-4350

UNITED STATES

robin@math.washington.edu

MATTHIAS HAMMERL

DEPARTMENT OF MATHEMATICS AND INFORMATICS

UNIVERSITY OF GREIFSWALD

WALTHER-RATHENAU-STR. 47

D-17489 GREIFSWALD

GERMANY

matthias.hammerl@uni-greifswald.de 


\title{
PACIFIC JOURNAL OF MATHEMATICS
}

Founded in 1951 by E. F. Beckenbach (1906-1982) and F. Wolf (1904-1989)

$$
\text { msp.org/pjm }
$$

\section{EDITORS}

\author{
Don Blasius (Managing Editor) \\ Department of Mathematics \\ University of California \\ Los Angeles, CA 90095-1555 \\ blasius@math.ucla.edu
}

\author{
Paul Balmer \\ Department of Mathematics \\ University of California \\ Los Angeles, CA 90095-1555 \\ balmer@math.ucla.edu \\ Robert Finn \\ Department of Mathematics \\ Stanford University \\ Stanford, CA 94305-2125 \\ finn@math.stanford.edu \\ Sorin Popa \\ Department of Mathematics \\ University of California \\ Los Angeles, CA 90095-1555 \\ popa@math.ucla.edu
}

\author{
Vyjayanthi Chari \\ Department of Mathematics \\ University of California \\ Riverside, CA 92521-0135 \\ chari@math.ucr.edu \\ Kefeng Liu \\ Department of Mathematics \\ University of California \\ Los Angeles, CA 90095-1555 \\ liu@math.ucla.edu \\ Igor Pak \\ Department of Mathematics \\ University of California \\ Los Angeles, CA 90095-1555 \\ pak.pjm@gmail.com \\ Paul Yang \\ Department of Mathematics \\ Princeton University \\ Princeton NJ 08544-1000 \\ yang@math.princeton.edu
}

\section{PRODUCTION}

Silvio Levy, Scientific Editor, production@msp.org

\section{SUPPORTING INSTITUTIONS}

ACADEMIA SINICA, TAIPEI

CALIFORNIA INST. OF TECHNOLOGY

STANFORD UNIVERSITY

UNIV. OF BRITISH COLUMBIA

UNIV. OF CALIFORNIA, BERKELEY

UNIV. OF CALIFORNIA, DAVIS

UNIV. OF CALIFORNIA, LOS ANGELES

UNIV. OF CALIFORNIA, RIVERSIDE

UNIV. OF CALIFORNIA, SAN DIEGO

UNIV. OF CALIF., SANTA BARBARA
KEIO UNIVERSITY

MATH. SCIENCES RESEARCH INSTITUTE

NEW MEXICO STATE UNIV.

OREGON STATE UNIV.
Daryl Cooper

Department of Mathematics

University of California

Santa Barbara, CA 93106-3080 cooper@math.ucsb.edu

Jiang-Hua Lu

Department of Mathematics

The University of Hong Kong

Pokfulam Rd., Hong Kong

jhlu@maths.hku.hk

$$
\text { Jie Qing }
$$

Department of Mathematics

University of California

Santa Cruz, CA 95064

qing@ cats.ucsc.edu

\author{
UNIV. OF CALIF., SANTA CRUZ \\ UNIV. OF MONTANA \\ UNIV. OF OREGON \\ UNIV. OF SOUTHERN CALIFORNIA \\ UNIV. OF UTAH \\ UNIV. OF WASHINGTON \\ WASHINGTON STATE UNIVERSITY
}

These supporting institutions contribute to the cost of publication of this Journal, but they are not owners or publishers and have no responsibility for its contents or policies.

See inside back cover or msp.org/pjm for submission instructions.

The subscription price for 2016 is US $\$ 440 /$ year for the electronic version, and \$600/year for print and electronic.

Subscriptions, requests for back issues and changes of subscriber address should be sent to Pacific Journal of Mathematics, P.O. Box 4163, Berkeley, CA 94704-0163, U.S.A. The Pacific Journal of Mathematics is indexed by Mathematical Reviews, Zentralblatt MATH, PASCAL CNRS Index, Referativnyi Zhurnal, Current Mathematical Publications and Web of Knowledge (Science Citation Index).

The Pacific Journal of Mathematics (ISSN 0030-8730) at the University of California, c/o Department of Mathematics, 798 Evans Hall \#3840, Berkeley, CA 94720-3840, is published twelve times a year. Periodical rate postage paid at Berkeley, CA 94704, and additional mailing offices. POSTMASTER: send address changes to Pacific Journal of Mathematics, P.O. Box 4163, Berkeley, CA 94704-0163.

PJM peer review and production are managed by EditFLOW ${ }^{\circledR}$ from Mathematical Sciences Publishers.

PUBLISHED BY

\section{I. mathematical sciences publishers}

nonprofit scientific publishing

http://msp.org/

(C) 2016 Mathematical Sciences Publishers 


\section{PACIFIC JOURNAL OF MATHEMATICS}

Volume $285 \quad$ No. $2 \quad$ December 2016

The $\mathrm{SU}(N)$ Casson-Lin invariants for links

HANS U. BODEN and ERIC HARPER

The SU(2) Casson-Lin invariant of the Hopf link

HANS U. BODEN and CHRISTOPHER M. HERALD

Commensurations and metric properties of Houghton's groups

José BuRILlo, SEAn Cleary, ARMANDo MaRTino and ClaAs

E. RÖVER

Conformal holonomy equals ambient holonomy

ANDREAS ČAP, A. RoD GOVER, C. RoBIN GRAHAM and

MATTHIAS HAMMERL

Nonorientable Lagrangian cobordisms between Legendrian knots

ORSOLA CAPOVILLA-SEARLE and LISA TRAYNOR

A strong multiplicity one theorem for $\mathrm{SL}_{2}$

JINGSONG CHAI and QING ZHANG

The Yamabe problem on noncompact CR manifolds

PAK TUNG Ho and SeONGTAG KIM

Isometry types of frame bundles

WOUTER VAN LIMBEEK

Bundles of spectra and algebraic K-theory

JOHN A. LIND

Hidden symmetries and commensurability of 2-bridge link complements

CHRISTIAN MiLlichaP and WILLIAM WORDEN

On seaweed subalgebras and meander graphs in type $C$

DMITRI I. PANYUSHEV and OKSANA S. YAKIMOVA

The genus filtration in the smooth concordance group 